\title{
Efectos de una intervención educativa sobre los niveles plasmáticos de LDL-colesterol en diabéticos tipo 2
}

\author{
Carlos Enrique Cabrera-Pivaral, MC, M en Educ, DC S, ${ }^{(1,2)}$ G uillermo González-Pérez, SoC, M en Demogr, D CS, (2)
} María Guadalupe Vega-López, TS, MSP, D ra en Soc, DCS, ${ }^{(2)}$ Mayarí Centeno-López, MC, M en Educ, DCS. ${ }^{(3)}$

\section{Cabrera-Pivaral CE, González-Pérez G, Vega-López MG, Centeno-López M. Efectos de una intervención educativa sobre los niveles plasmáticos \\ de LDL-colesterol en diabéticos tipo 2. Salud Publica Mex 2001;43:556-562.} El texto completo en inglés de este artículo está disponible en: http://www.insp.mx/salud/index.html

\section{Resumen}

Objetivo. D emostrar las ventajas de una intervención educativa en el control del colesterol de baja densidad (LDL colesterol) en el paciente diabético tipo 2. Material y métodos. Se realizó un estudio cuasi experimental con asignación aleato ria de dos grupos de pacientes diabéticos: un grupo de experimento y un grupo control. El grupo de experimento se integró con 25 diabéticos tipo 2 y el control con 24. La inter vención educativa-participativa se organizó mediante el proceso de reflexión-acción. Se efectuaron mediciones de los niveles séricos del LDL colesterol basales y mensuales durante los nueve meses de la intervención educativa. Los grupos fueron controlados tomando en cuenta edad y sexo. El análisis estadístico se efectuó con el estadígrafo deW ilcoxon para variables ordinales y grupos relacionados. Resultados El grupo que recibe la intervención educativa participativa logra un valor promedio de 148.4+/-21.3, en comparación con el grupo control (185+/-24.1) en la medición posterior del colesterol LDL $(p \leq 0.05)$. Conclusiones La intervención educativa participativa, mediante la promoción de un nuevo estilo de vida en el paciente diabético del tipo 2, contribuye a mejorar el nivel de control metabólico del LDL colesterol. El texto completo en inglés de este artículo está disponible en: http:/ /www.insp.mx/salud/index.html

Palabras clave: intervención; educación en salud; diabetes mellitus no insulino-dependiente; colesterol LD L; México

\author{
Cabrera-Pivaral CE, González-Pérez G, \\ Vega-López MG, Centeno-López M. \\ Effects of an educational intervention \\ on plasma levels \\ of LDL cholesterol in type-2 diabetics. \\ Salud Publica Mex 2001;43:556-562. \\ The English version of this paper \\ is available at: http://www.insp.mx/salud/index.html
}

\begin{abstract}
A bstract
Objective. To prove the benefit of an educational intervention for controlling LDL cholesterol levels in LDL cholesterol. Material and Methods. A quasi-experimental study was conducted; diabetic patients were randomly allocated to an experimental and a control group. The experimental group consisted of 25 patients and the control group of 24 patients. The educational intervention was organized through a reflection-action process. LDL cholesterol levels were measured at baseline and monthly during the nine months of the study.The groups were controlled for age and sex. Statistical analysis included W ilcoxon's test for ordinal variables. Results. The intervention gro up had a mean value of LDL cholesterol of 148.4 +/-21.3, compared to 185 $+/-24.1$ in the control group $(p \leq 0.05)$. Conclusions. The participative educational intervention contributed to improving the levels of LDL cholesterol, by promoting a lifestyle change in type-2 diabetic patients. The English version of this paper is available at: http://www.insp.mx/salud/index.html
\end{abstract}

Key words: intervention; health education; diabetes mellitus, non-isulin-dependent; LD L cholesterol; Mexico

(1) Unidad de Investigación en Epidemiología C línica, Hospital de Especialidades, Centro Médico N acional de 0 ccidente, Instituto Mexicano del Seguro Social, Guadalajara, México.

(2) Centro de Estudios en Salud, Población y Desarrollo Humano, Departamento de Ciencias Sociales, Centro Universitario de Ciencias de la Salud, Universidad de Guadalajara, Guadalajara, México.

(3) Educación e Investigación Médica, H ospital General Regional 45, IMSS.

Fecha de recibido: $\mathbf{2 5}$ de octubre de $\mathbf{2 0 0 0} \bullet$ Fecha de aprobado: 22 de agosto de 2001

Solicitud de sobretiros: MC . C arlos Enrique C abrera-Pivaral. San Juan Bosco 3782, colonia C hapalita, Guadalajara, Jalisco, México. Correo electrónico: carlose @ mail.udg.mx. 
os estilos de vida no saludables se han relacionado con las alteraciones del perfil de lípidos séricos, y por lo tanto, con la ateroesclerosis, reconocida ésta como la principal causa de los síndromes coronarios. De éstos, el infarto al miocardio y la angina inestable son las principales causas de morbilidad y mortalidad en el mundo industrializado ${ }^{1,2}$ Ambas patologías son consideradas prevenibles mediante actividades de promoción y educación para la salud. ${ }^{2}$

La educación para la salud es un proceso social que se alimenta del cúmulo de teorías y métodos que ofrecen las ciencias médicas y las de la educación, ${ }^{3}$ con las cuales se analiza el proceso de la salud-enfermedad. El propósito de la misma es informar y motivar a la población a adoptar y mantener prácticas y estilos de vida saludables, además de propiciar cambios ambientales, dirigir la formación de recursos humanos y la investigación en su propio campo. ${ }^{4}$

Como proceso, la educación para la salud se instrumenta a partir de la reflexión-acción; el educador y el educando efectúan un razonamiento acerca de los estilos de vida saludables mediante la entrevista y la acción, organizadas a partir de reconocer sus ventajas y aciertos. ${ }^{4}$

La promoción de la salud y la medicina preventiva incorporan factores culturales y comunicativos que condicionan la participación activa de los pacientes diabéticos 2, frente a la toma de decisiones sobre estilos de vida saludables.

El perfil del paciente con diabetes mellitus tipo 2, se caracteriza por obesidad exógena y malos hábitos alimenticios que se relacionan con un ambiente externo que no participa en informar sobre su salud, ni motiva para adoptar y mantener hábitos y estilos de vida saludables. Los hábitos alimenticios inadecuados ocasionan trastornos en el perfil metabólico de los lípidos séricos. El 20\% de los pacientes diabéticos tiene alteraciones en el perfil de los lípidos ${ }^{1}$ y esto es un factor de riesgo para los trastornos cardiovasculares. ${ }^{5,6,7}$

La aplicación de un plan educativo tipo participativo, basado en la reflexión acción sobre aspectos de la nutrición humana en el paciente diabético 2, será el camino para el control del LDL colesterol sérico, y a largo plazo se traducirá en la disminución del riesgo cardiovascular. $^{8}$

\section{Material y métodos}

Se realizó un estudio cuasi experimental en 49 pacientes diabéticos tipo 2 sin tratamiento medicamentoso, con valores séricos de LDL colesterol altos, e interesados en participar en un proyecto de intervención educativa durante un periodo de nueve meses. Todos ellos eran usuarios de los servicios de salud de Medicina Familiar en el Instituto Mexicano del Seguro Social en Guadalajara, Jalisco, México, entre 1999 y 2000. El propósito fue demostrar las ventajas de la educación participativa en el control del LDL colesterol sérico en pacientes diabéticos tipo 2 con obesidad (Indice de Masa Corporal $(\mathrm{IMC})>27 \mathrm{y}<35$ ).

Los pacientes a estudiar fueron convocados mediante una asamblea en la Unidad de Medicina Familiar. De los asistentes, aceptaron participar $60 \%$, los cuales fueron asignados en forma aleatoria a dos grupos, uno para recibir la educación participativa (grupo experimento), y el otro, la educación tradicional (grupo control), para su problema de salud. ${ }^{9}$ Se tomaron en cuenta la edad y el sexo, y se exploraron la escolaridad y los años de evolución de la diabetes 2, sin encontrar diferencias estadísticas entre ambos grupos antes de la intervención.

El grupo experimental se formó de 25 pacientes y el control de 24, quienes cubrieron un mínimo de $90 \%$ de asistencia (32 sesiones).

El programa educativo participativo se desarrolló una vez por semana durante nueve meses, con una duración máxima de dos horas cada sesión, coordinado por una trabajadora social y un nutriólogo adiestrado en la metodología participativa. Los contenidos temáticos abordaron conceptos y definiciones de la diabetes mellitus 2; anatomía, fisiología, psicología y comportamiento humano, complicaciones agudas y crónicas, nutrición básica y aplicada, grupos básicos de los alimentos, nutrimentos, dietas, autocontrol, ejercicio, higiene y apoyo familiar. Los contenidos temáticos se organizaron para un momento reflexivo, con duración aproximada de 1.4 horas, y para un momento de acción (0.6 hora) con desarrollo de talleres, trabajo en equipo y la discusión de las experiencias de aprendizaje con la guía de comportamiento dietético.

El proceso educativo participativo se caracterizó por: a) el interés en los procesos y efectos; $b$ ) la consideración del educando como un sujeto crítico y creativo; c) la búsqueda del desarrollo de las habilidades y capacidades naturales del sujeto para transformar la realidad individual y social; d) la comunicación a través del diálogo, el intercambio de ideas y la comparación de acciones entre los participantes; e) la utilización de técnicas de educación y participación comunitarias y f) la incorporación de la relación de los objetivos informativos y formativos. ${ }^{10}$

La reflexión consistió en un análisis crítico por parte de los participantes sobre las necesidades sentidas y expresadas con relación a su control metabólico mediante técnicas de educación participativa que fa- 
vorecieron la integración, comunicación y análisis general. La acción se caracterizó por el proceso de planeación y organización de actividades de autocuidado a través del equipo comunitario, con el fin de modificar los hábitos dietéticos y lograr el control metabólico. Los talleres en aula de aprendizaje favorecieron la construcción de una guía de comportamiento higiénico-dietético.

La guía de comportamiento surgió después de un taller con una dinámica educativa denominada lluvia de ideas, la cual permitió integrar un listado de hábitos a observar durante la vida cotidiana de los pacientes.

En general, se desarrollaron cuatro niveles de abordaje:

1. El autodiagnóstico, que se constituyó mediante el conocimiento de la realidad en la cual el paciente diabético vive cotidianamente con su enfermedad y sus hábitos de alimentación.

2. La teorización, basada en los conceptos operativos de diabetes, nutrición y hábitos de alimentación.

3. La práctica, que consistió en talleres de aplicación de las leyes de la alimentación y la nutrición.

4. La autoevaluación realizada en dos etapas: la relacionada con el apego a la guía de cotejo de los hábitos de comportamiento dietético, y la del control metabólico logrado durante el mes.

La intervención educativa tradicional en el grupo control se caracterizó por las estrategias comunicativas de tipo informativo, con el apoyo de medios audio-visuales que permitían el intercambio de ideas. Se desarrolló una vez por semana durante nueve meses. Los contenidos temáticos fueron primero expuestos mediante conferencias magistrales y demostraciones con una duración aproximada de 1.2 horas cada una, para posteriormente analizar las experiencias con las guías de comportamiento dietético.

El propósito fue mantener el orden del proceso educativo, en el cual el docente informa al educando, reconociendo la necesidad de ciertas mejoras en el proceso y en donde los contenidos educativos son asignados con base en una jerarquización a priori efectuada por el docente.

Los temas y contenidos del curso fueron los mismos que se desarrollaron en el grupo de experimento. El aprendizaje en el grupo de metodología tradicional inició con la recepción de conocimientos y memorización de los datos más importantes, como los nutrimentos, las calorías, el control metabólico y los hábitos higiénicos. La construcción y asignación de la lista de cotejo de los hábitos de comportamiento a seguir se elaboró con base en los contenidos y temas desarrollados por el profesor coordinador.

\section{Técnicas de laboratorio}

Se efectuó mensualmente la medición del colesterol LDL y glucosa sanguíneos en ayunas, por duplicado en una unidad hospitalaria, utilizando el método enzimático. El control de calidad se realizó según lineamientos de la Organización Mundial de la Salud (OMS), con curvas de calibración y variancia en condiciones óptimas. Para determinar la medición se utilizó el promedio del valor del LDL colesterol en cada paciente de los últimos tres meses de la intervención.

\section{A nálisis estadístico}

Los resultados se expresan utilizando la media y desviación estándar. Además, se clasifica el nivel LDL colesterol en malo (> $200 \mathrm{mg} / \mathrm{dl})$, regular $(150-200$ $\mathrm{mg} / \mathrm{dl})$, y bueno $(<150 \mathrm{mg} / \mathrm{dl})$. Para la comparación entre los grupos se llevó a cabo la prueba de rangos señalados y pares igualados de Wilcoxon para grupos relacionados, de acuerdo con la variable ordinal y la $t$ de Students para grupos relacionados.

El protocolo fue previamente autorizado por el Comité de Investigación y de Ética de la Institución, y se obtuvo además el consentimiento informado de los participantes según el artículo 17 de la Ley General de Salud en materia de investigación en seres humanos.

\section{Resultados}

\section{Datos generales de los grupos de estudio}

El grupo experimental se conformó con 13 pacientes de sexo masculino (52.0\%), y $12(48.0 \%)$ del sexo femenino, y el grupo control con 11 de sexo masculino $(45.8 \%)$, y $13(54.2 \%)$ del sexo femenino $\left(\chi^{2}=0.18\right.$; $p=0.67)$. La edad en el grupo experimento fue de $58.1 \pm 12.4$ años y en el grupo control de $57.8 \pm 8.7(t=0.68$; $p=0.50$ ). Con respecto a la escolaridad se observó que la moda en el grupo de experimento fue la primaria completa en $44.0 \%$ de los casos y para el grupo de control fue la secundaria completa en $41.7 \%$ de los pacientes $(t=2.79 ; p=0.09)$. El IMC entre ambos grupos demuestra una homogeneidad sin diferencia estadística $(t=0.47 ; p=0.7)$. Con relación a los años de evolución de la diabetes mellitus para el grupo experimental fue de $8.8 \pm 9.0$ años, y para el grupo control de $11.3 \pm 11.8$ $(t=0.81 ; p=0.57)$, sin mostrar diferencias en la medición inicial y final entre los grupos de estudio $(p=0.57)$. 


\section{Grupo de control}

Los valores de LDL colesterol antes de la intervención educativa en el grupo control muestran un promedio de $195 \mathrm{mg} / \mathrm{dl}$, desviación estándar de 30.07 y distribución bajo la curva normal. La medición ordinal se distribuye entre 10 pacientes $(41.3 \%)$ con mal control (>200 mg/dl ), 12 pacientes (50\%) con regular control $(150-200 \mathrm{mg} / \mathrm{dl})$ y $2(8.3 \%)$ con buen control $(<150$ $\mathrm{mg} / \mathrm{dl}$ ) (cuadro I). Después de la intervención educativa en este grupo de estudio se observa un promedio de $185 \mathrm{ml} / \mathrm{dl}$ con desviación estándar de 24.13 y al considerar la medición de la variable ordinal se observa a cuatro pacientes en la medición de malo, 19 en la medición de regular y uno en la medición de bueno. $\mathrm{Al}$ relacionar los valores del pre y del post se muestra una diferencia estadística ( $p=0.01$ ) (cuadro II).

\section{Grupo de experimento}

En el grupo de experimento antes de la intervención el promedio fue de $199.2 \mathrm{mg} / \mathrm{dl}$, desviación estándar de 25.3 y distribución bajo la curva normal. El indicador ordinal ubicó a 12 pacientes en categoría de malo, a 12 en regular y a uno en bueno.

El grupo de experimento después de la intervención muestra una media de $148.4 \mathrm{mg} / \mathrm{dl}$ con 21.3 de

Cuadro I

VALORES de LDL COLESTEROL SEgún GRUPO de ESTUDIO, ANTES Y DESPUÉS DE LA INTERVENCIÓN educativa. Guadalajara, México, 1999-2000

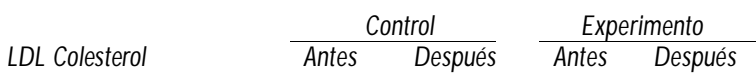

\begin{tabular}{lcccc} 
Malo $>200 \mathrm{mg} / \mathrm{dl}$ & 10 & 4 & 12 & 0 \\
\hline Regular $150-200 \mathrm{mg} / \mathrm{dl}$ & 12 & 19 & 12 & 13 \\
\hline Bueno $<150 \mathrm{mg} / \mathrm{dl}$ & 2 & 1 & 1 & 12 \\
\hline Total & 24 & 24 & 25 & 25
\end{tabular}

W ilcoxon:17.6; $p=0.008$

Cuadro II

Colesterol LDL en los grupos de estudio. Guadalajara, México, 1999-2000

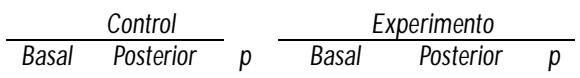

$\begin{array}{lllllll}\text { Colesterol-LDL* } & 195 \pm 30 & 185 \pm 24.1 & 0.01 & 199.2 \pm 25.3 & 148.4 \pm 21.3 & 0.05\end{array}$

$\cdot \mathrm{mg} / \mathrm{dl}$ desviación estándar; el indicador ordinal demuestra que ningún paciente diabético ocupa el nivel de malo, 13 están en el nivel de regular y 12 en el nivel de bueno. Al relacionar los valores de LDL colesterol entre la pre y la post intervención existe una disminución de los mismos y se presenta una diferencia estadística significativa $(p=0.007)$ (cuadro I y II).

\section{Comparación del efecto según grupo de estudio}

Al efectuar un análisis estadístico entre el grupo de experimento y el de control, antes de la intervención educativa, no se observó una diferencia estadística significativa. ( $p=>0.05)$.

El análisis estadístico después de la intervención educativa entre el grupo de experimento y el grupo control, muestra una diferencia estadísticamente significativa $(p=<0.05)$ a favor del grupo de experimento.

Se observa que la media del LDL colesterol por grupo etáreo aumenta proporcionalmente con la edad, sin mostrar una diferencia estadística que relacione a ésta con la disminución del valor de LDL colesterol en el grupo de experimento (cuadro III).

$\mathrm{Al}$ relacionar las mediciones finales del colesterol LDL en el grupo control y en el de experimento, según el tiempo de evolución de la diabetes, no se identifica una relación estadística que explique la diferencia de los valores séricos finales.

Al efectuar una correlación entre el IMC y el LDL colesterol se identifica una influencia entre ambas, sugiriendo que la relación de la medida antropométrica sobre los valores séricos del LDL colesterol con el valor $p$ advertido indica que es poca la probabilidad de que la magnitud de la correlación informada ocurra por el azar (cuadro IV).

Con respecto a los valores de glucosa en sangre en ayunas, según grupo de experimento y de control antes-después de la intervención, se observa una homogeneidad en los valores iniciales $(210.9 \mathrm{mg} / \mathrm{dl}$ con

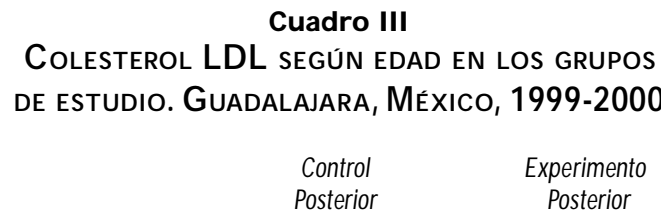

\begin{tabular}{|c|c|c|}
\hline$<50$ años & $178 \pm 25.5$ & $143.1 \pm 21.1$ \\
\hline $50-59$ & $184 \pm 23.8$ & $147.1 \pm 22.2$ \\
\hline$>60$ & $193 \pm 22.7$ & $155 \pm 20.6$ \\
\hline
\end{tabular}

(t: $0.68 ; p: 0.5)$ 
\pm 43.9 y 221.6 con $\pm 83.2 ; p=>0.05$ ). Al finalizar la intervención se muestra una diferencia estadística a favor del grupo de experimento (147.6 con \pm 32.8 y $182.8 \pm 48.2$ ) (cuadro V).

\section{Discusión}

La modificación de comportamiento del paciente diabético tipo 2 como resultado de la intervención comunicativo-educativa que condiciona sus hábitos y destrezas, tiene como objetivo alcanzar su control metabólico normal del LDL colesterol, y prevenir los riesgos y complicaciones de la elevación de sus niveles. ${ }^{3}$ No descartamos el papel fundamental de otros factores aparte del educativo, sino que enfatizamos éste como importante para mejorar las condiciones de vida de los pacientes diabéticos. ${ }^{11}$

Para el logro del control metabólico de los lípidos séricos en el paciente diabético, los trabajadores de la salud, incluido el enfermo, desarrollan un proceso de reflexión-acción en conjunto para estructurar un plan de manejo de los principales factores de riesgo: el control no sólo de un nivel adecuado del LDL colesterol, sino también de los valores del IMC más cercanos a los valores aceptados, valores que si bien en el estudio no logran la normalidad, muestran disminuciones favorables para la salud del paciente, especialmente en el grupo que recibe la intervención participativa.

\section{Cuadro IV}

Correlación entre Indice de Masa Corporal

y Colesterol LDL en los grupos de estudio. Guadalajara, México, 1999-2000

\begin{tabular}{lccccccc} 
& \multicolumn{3}{c}{ Control } & & \multicolumn{3}{c}{ Experimento } \\
\cline { 2 - 3 } & Basal & Posterior & $p$ & & Basal & Posterior & $p$ \\
IMC & $34.1 \pm 4.2$ & $32.4 \pm 2.4$ & 0.11 & & $34.8 \pm 3.6$ & $29.4 \pm 2.3<0.05$ \\
$(r=0.69 ; p=0.05)$ & & & & & &
\end{tabular}

\section{Cuadro V \\ Glucosa en SANGRe en AYUNAS SEgún GRUPo de estudio. Guadalajara, México, 1999-2000}

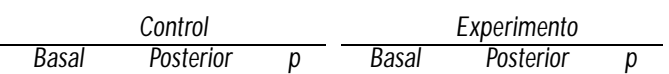

Glucosa

$\mathrm{mg} / \mathrm{dl} \quad 221.6 \pm 83.2 \quad / 182.8 \pm 48.2 \quad />0.11 \quad 210.9 \pm 43.9 \quad / 147.6 \pm 32.8 \quad /<0.05$

$(t=10.45 ; p=0.001)$
Según los valores normales de LDL colesterol, ${ }^{1,12,13}$ la tercera parte de los diabéticos tipo 2 estudiados logran al final de la intervención el nivel de bueno; de ellos, $16 \%$ corresponden al grupo de control y $50 \%$ al grupo de experimento.

Keller ${ }^{12}$ y Goldman ${ }^{13}$ demuestran la disminución de colesterol y lípidos séricos mediante un proyecto educativo nutricional de tipo tradicional en pacientes con diabetes tipo 2. Sin embargo, hay que reconocer las limitaciones de los proyectos tradicionales que en su mayoría se caracterizan por ser más informativos que formativos, de tipo exógeno, en donde se trata al educando como un objeto mediante una relación de tipo autoritaria. El grupo control recibió una estrategia comunicativa tradicional de tipo informativa y logró algunos beneficios importantes en la posmedición del proyecto. Es importante destacar que la educación participativa forma al paciente diabético, convirtiéndolo en un ser más reflexivo y comprometido para mejorar sus condiciones de salud y de vida.

Herd señala que mediante el logro de una disminución igual o mayor al 20\% del LDL colesterol se asegura la disminución en la severidad de la ateroesclerosis, objetivo alcanzado por el grupo de experimento que logra disminuir $25.7 \%$ de su medición inicial. ${ }^{14,15}$ Sin embargo, y a pesar de que el proyecto no buscó como efecto principal prevenir lesiones clínicas, sus resultados deberán ser motivo de nuevas hipótesis para proyectos futuros, que desde la perspectiva de la educación y la cultura en salud logren vincular el beneficio clínico.

Hershowitz ${ }^{16}$ demostró que mediante técnicas de motivación, participación y autocuidado es más fácil desarrollar esquemas de tipo formativo con los pacientes diabéticos que se traduzcan en el control de factores de riesgo, como lo es el cardiovascular. ${ }^{17} \mathrm{La}$ intervención educativa favoreció el autocuidado y la conciencia social en salud, fomentando el área de la educación para la salud y de la psicología social, al utilizar el co-monitoreo, el co-registro, la co-evaluación y el co-reforzamiento, con la finalidad de que el paciente se apegue al tratamiento y mejore así sus condiciones de salud y de vida.

En los países en desarrollo existe hoy acuerdo general sobre la importancia de los estilos de vida en la causalidad de los problemas de salud, así como también en la importancia del cumplimiento de las prescripciones y recomendaciones médicas. ${ }^{18}$ Todos los países coinciden en poner en marcha programas de educación sanitaria con el fin de prevenir la enfermedad y sus complicaciones mediante la modificación de los comportamientos humanos. El presente estudio ofrece una metodología educativa útil que da respues- 
ta al cómo acercarse a los diabéticos para que participen en su autocuidado y se acerquen a los valores aceptados de normalidad.

Compartimos la afirmación de Ulrich, ${ }^{19}$ en cuanto a que el objetivo de la educación sanitaria es estimular y desarrollar al paciente diabético 2, sin descuidar lo que Freire ${ }^{20}$ menciona: al hombre como esencialmente crítico para la transformación de la naturaleza en un beneficio razonado y creativo.

Steckler ${ }^{21}$ describe la influencia e impacto de las intervenciones educativas en salud mediante la vinculación de la teoría con la práctica, y define las estrategias de intervención enfatizando que los proyectos educativos en grupo generan más ventajas en el aprendizaje, ${ }^{6,8,13}$ como lo establecido en este estudio, que valora el proceso grupal sobre el proceso individual, de tipo informativo enciclopedista.

El tamaño muestral no permite establecer inferencias estadísticas; sin embargo, se logra observar resultados valiosos para grupos con características similares; motivo por el cual se continuará con proyectos que permitan sustentar la inferencia estadística en poblaciones con características similares.

Durante el proceso de aprendizaje existen factores psicológicos, como la negación de la enfermedad, estados de ansiedad, de desánimo, de apatía y la resistencia a "ser enseñados", así como factores sociales: trabajo, obligaciones familiares, los prejuicios ante la dolencia, que unidos a la desinformación y no formación limitan el interés y la posibilidad de aprendizaje de los sujetos con diabetes. Estos factores no medidos en el reporte son manifiestos durante los espacios de la enseñanza-aprendizaje, especialmente en el grupo de intervención participativa.

Leino $^{6}$ analiza cómo el nivel de escolaridad entre los diabéticos tipo 2 define sus estilos de vida, además de caracterizar el control metabólico de su enfermedad. En nuestro estudio la escolaridad no mostró diferencia en los grupos de estudio, pero seguramente condiciona la información y la formación de hábitos en salud, factores básicos para planear programas de promoción de la salud. ${ }^{4,5,22}$

El beneficio de recibir una orientación educativa temprana, adecuada y oportuna para el cuidado y autocontrol de la dolencia ${ }^{22}$ ha sido un acuerdo aceptado por instituciones de servicios de salud, producto de reconocer que el conocimiento que las personas sanas tienen de la diabetes, incluso las que la padecen, suele ser precario y frecuentemente sesgado y pleno de tabúes, errores y distorsiones. ${ }^{22,23}$ Esto limita la capacidad del enfermo para tomar decisiones sobre la importancia de su participación activa en el control y, por lo tanto, en la asistencia a programas educativos.

Finalmente, la intervención educativa participativa, mediante la reflexión-acción del paciente diabético 2 , ofrece mejores beneficios en el control del LDL colesterol que la intervención tradicional.

\section{Referencias}

1. Secretaría de Salud, Dirección General de Epidemiología, Encuesta N acional de Enfermedades C rónicas. 2a ed. México D.F.: SSA, 1995:31-35.

2. Shepherd J, Weissberg P. Time to treat: Beyond survival, preventing recurrence of acute coronary syndromes. Atherosclerosis 1999;147 (Suppl):3-10.

3. N ovoa-Menchaca A. C urrículum y Plan de estudios de la Maestría en Educación para la Salud. U niversidad de Guadalajara. Guadalajara, México 1984:5.

4. Salleras-San Martín L. Educación Sanitaria. Madrid: Díaz de Santos, 1990:20-26.

5. Giampaoli S, Menotti A, Amici R, Cigna G, D ima F, Feskens E et al. Prevalence of some cardiovascular risk factors in a sample of the aged population. Cardiologia 1992;37(12):865-870.

6. Leino M, Raitakari OT, Porkka KV, Taimela S, Viikar JS. Associations of education with cardiovascular risk factors in young adults. Int J Epidemiol 1999;28(4):667-675.

7. Howard B, Robbins D, Sievers M, Lee E, Rhoades D et al. LD L Cholesterol as a strong predictor of coronary heart disease in diabetic individuals with insuline resistance and low LDL:The strong heart study.Arterioscler Thromb Vasc Biol 2000; 20(3):830-835.

8. Schwandt P, Geiss HC, Ritter MM, U blacker C, Parhofer KG, $O$ tto $C$ et al.The prevention education program.A prospective study of the efficacy of family oriented life style modification in the reduction of cardiovascular risk and disease: Design and baseline data. J Clin Epidemiol 1999;52(8):791-800.

9. C ampbell D, Stanley J. D iseños experimentales y cuasiexperimentales en la investigación social. Buenos Aires:A morrortu, 1993:24-48.

10. Kaplun M. El comunicador popular. Buenos Aires Humanitas, 1990: $12-22$.

11. Dowse GK, Gareeboo H,Alberti KG , Zimmet P,Tuomilehto J, Purran A et al. Changes in population cholesterol concentrations and other cardiovascular risk factor levels after five years of the non communicable disease intervention programme in Mauritius. Mauritius non communicable disease study group. BMJ 1995;311(7015):1255-1259.

12. Keller U. Health education and nutrition. Schweitz Med W 1991; 121(27):1014.

13. Goldman L, Coxson P, Hunink MG, Goldman PA, Tosteson AN, Mittleman $M$ et al. The relative influence of secondary versus primary prevention using the national cholesterol education program adult treatment panel II guidelines. J A m Coll C ardiol 1999;34(3):768-776.

14. Herd A. Relation of clinical benefit to metabolic effects in lipid-lowering therapy.Am J C ardiol 1998;82:22M-25M.

15. Werner RM, Pearson TA. LD L cholesterol: A risk factor for coronary artery disease from epidemiology to clinical trials. Can J Cardiol 1998;14:suppl B:3B-10B.

16. Hershowitz R. Education and behavior in diabetes care. Diabetic Med 1990;7(7):633. 
17. Forrest KY, Becker DJ,W olfson SK, O rchard TJ.Are predictors of coronary heart disease and lower extremity arterial disease in type 1 diabetes the same.Arteriosclerosis 2000;148(1):159-169.

18. Marti B, Dai S, Rickenbach M,W ietlisbachV, Bucher C, Barazzoni F et al. Total cholesterol, HDL-cholesterol and blood presure in relation to life style. Schweiz Med W ochenschr 1990;120(51):1976-1988.

19. U Irich $A$. The integration of health education to patient care. 1984;35:120-125.
20. Freire P. Extensión o comunicación. La concientización en el medio rural. México, D.F: Siglo XXI, 1981:120-132.

21. Steckler A,Allegrante JP,A Itman D. Health education intervention strategies. Health Educ Q 1995;22(3):307-328.

22. García-Viveros M. Salud comunitaria y promoción de la salud. Disponible en: www.iceps.es/salcomun.htm> 2001:1-18.

23. Sweeney K, G ray D P, Evans P, Steele R.The doctrine of early intervention. BMJ 1996;313(7065):1097. 